\title{
Measurement of the Orientation Distribution of Grains in Titanium Sheets by Etch Pit Method*
}

\author{
By Yonosuke Murayama**, Kazuo Obara** and Eihachiro Tanaka***
}

\begin{abstract}
In this experiment, the procedure to form oriented etch pits on a commercially pure titanium sheet was presented, and the feature of the orientation distribution of grains, containing the orientation relation between neighboring grains, was analyzed by the etch pit method. The shape of the etch pit is coincident with that introduced from the rotation of the 14-hedral model formed by the $\{0001\}$ planes and the $\{10 \overline{1} 1\}$ planes. Shape parameters, $R_{c}, R_{p}$ and $R_{a}$, have the following relation. $R_{c}: R_{p}=1: 1.4\left(R_{a}=R_{p} /\right.$ $\sin (61.4 \pi / 180)=1.6)$.

Using such oriented etch pits, the feature of the orientation distribution in two differently textured titanium sheets were examined. In the sheets cross rolled at $873 \mathrm{~K}, 61 \%$ of the grains have the c-axis normal to the sheet plane. The preferred orientation is (0001)[1010] and the number of grains with such an orientation are $23.1 \%$. The frequency of the orientation difference of the c-axis between neighboring grains with angles between 0 and $\pi / 18$ is over $80 \%$. In the sheets rolled unidirectionally at $1173 \mathrm{~K}$, the grains with the c-axis parallel to the sheet plane are $50 \%$. The grains with the orientation in the range from $(10 \overline{1} 0)[11 \overline{2} 5]$ to $(10 \overline{1} 0[11 \overline{2} 17]$ are $12.5 \%$ in number. The preferred orientation is ranged from $(10 \overline{1} 0)[11 \overline{2} 5]$ to $(10 \overline{1} 0)[11 \overline{2} 17]$. The orientation difference of the c-axis between neighboring grains is distributed broadly in the whole range from 0 to $\pi / 2$, so that this distribution is extremely different from that of the sheets cross rolled at $873 \mathrm{~K}$.
\end{abstract}

(Received June 27, 1986)

Keywords: etch pit method, polyhedral model, texture, titanium, relation of the orientation between neighboring grains

\section{Introduction}

The cold rolled and recrystalization texture of pure titanium was first elucidated by Clark, $\mathrm{Jr}^{(1)}$. Since then, many reports on this problem have been published together with improvement of the measurement methods ${ }^{(2)}$. In most instances, the basal poles are titled about $7 \pi$ / 36 from the sheet normal direction toward the transverse direction, although the preferred orientation parallel to the rolling direction varies depending on the annealing temperature.

Since Hatch indicated that a $\mathrm{Ti}-4 \mathrm{Al}$ alloy with the basal texture showed the greatest degree of texture hardening ${ }^{(3)}$, it has been tried to control the texture by the condition of rolling and thermal treatment ${ }^{(4)}$. Also in pure

* This paper was originally published in Japanese in $\mathrm{J}$. Japan Inst. Metals, 49 (1985), 759.

** The Research Institute for Iron, Steel and Other Metals, Tohoku University, Sendai 980, Japan.

*** Professor Emeritus, Tohoku University. titanium, the position with maximum density of the basal pole can be varied from the sheet normal direction to the transverse direction by changing temperatures of rolling and annealing. Considerable work on the texture control of titanium and it's alloys has been done in connection with deformability or texture hardening. In these cases, it is a main subject to confirm the relationship between the major preferred orientation deduced from the pole figure and macroscopic anisotropy of plastic properties.

However, information on the orientation distribution of individual grains comprising the aggregate is needful, in order to evaluate some microscopic phenomena such as the surface roughness and ridging during forming ${ }^{(5)-(7)}$.

The etch pit method is one of the most convenient methods to measure the orientation of individual grains in a polycrystalline aggregate, if an adequate etchant is found. Although a case in which etch pits were produced on a titanium was known in literature ${ }^{(8)}$, no case in which oriented etch pits were produced and 
correlated with the orientation of grains was found.

In the present experiment, commercially pure titanium sheets were used. The etching procedure was shown and oriented etch pits were correlated with the orientation of grains by the polyhedral model. Then, the orientation distribution of grains in two differently textured titanium sheets was analyzed by the etch pit model.

\section{Procedure}

Table 1 shows the chemical composition of as-received commercially pure titanium sheets used in this experiment. The mechanical properties and the deformation mechanism of pure titanium vary with the amount of interstitial elements. The as-received hot rolled sheets used in this experiment contain about $0.1 \%$ oxygen.

Table 2 shows the etching procedure.

Table 1 Chemical composition of as-received $\mathrm{Ti}$ sheet in mass $\%$.

\begin{tabular}{ccccc}
\hline $\mathrm{N}$ & $\mathrm{C}$ & $\mathrm{H}$ & $\mathrm{Fe}$ & $\mathrm{O}$ \\
\hline$<0.001$ & 0.005 & 0.0006 & 0.035 & 0.099 \\
\hline \hline
\end{tabular}

Table 2 Etching procedure.

1. Mechanical polishing by 1000 emergy paper.

2. Electrolytic polishing.

Solution: $\mathrm{CH}_{3} \mathrm{OH} 300 \mathrm{cc}, \mathrm{N}-\mathrm{CH}_{3}\left(\mathrm{CH}_{2}\right)_{3} \mathrm{OH} 170 \mathrm{cc}$,

Voltage: $40 \mathrm{~V}$. $\mathrm{HClO}_{4} 30 \mathrm{cc}$.

Temperature: about $-65^{\circ} \mathrm{C}$.

Time: $240 \mathrm{~s}$.

3. Etching.

Solution: $\mathrm{C}_{3} \mathrm{H}_{6} \mathrm{O}_{3} 30 \mathrm{cc}, \mathrm{HNO}_{3} 10 \mathrm{cc}$ and $\mathrm{HF} 10 \mathrm{cc}$.

Temperature: room temperature.

Time: $30 \mathrm{~s}$.

4. Electrolytic etching.

Solution: $\mathrm{C}_{2} \mathrm{H}_{5} \mathrm{OH} 90 \mathrm{cc}, \mathrm{N}-\mathrm{CH}_{3}\left(\mathrm{CH}_{2}\right)_{3} \mathrm{OH} 10 \mathrm{cc}$.

Voltage: $20 \mathrm{~V}$.

$\mathrm{AlCl}_{2} 6 \mathrm{~g}$ and $\mathrm{ZnCl}_{2} 25 \mathrm{~g}$.

Temperature: room temperature.

Time: $60 \mathrm{~s}$.

5. Etching.

Solution: $\mathrm{H}_{2} \mathrm{O} 100 \mathrm{cc}, \mathrm{HF} 1 \mathrm{cc}$ and $\mathrm{HNO}_{3} 2 \mathrm{cc}$.

Temperature: room temperature.

Time: $45 \mathrm{~s}$.
Although there was a case in which etch pits were produced by electrolytic etching, No. $4{ }^{\left({ }^{(8)}\right.}$ a deep violet passive film was formed and the shape of etch pits was indistinct in this step, according to our experience. The shape of etch pits became sharp and was able to be correlated with the orientation of grains by etching, No. 5, after electrolytic etching, No. 4.

In order to correlate such etch pits with the crystallographic orientation, the orientation of coarse grains in titanium sheets formed by the strain annealed method was measured by the micro-Laue method. Then, the shape of oriented etch pits was compared with the figures of etch pits models, just as Kinoshita et $a l$. introduced for an austenitic stainless steel ${ }^{(9)}$, and the most appropriate polyhedral model was chosen.

These oriented etch pits and etch pit models were also applied to the analysis of textured sheets. The recrystalization texture of pure titanium sheets varies depending on the prior texture, the rolling temperature and the heat treatment ${ }^{(4)}$. Two kinds of recrystalization textured sheets were used in this experiment. One had the basal texture, the other the basal plane normal to the transverse direction. The former was cross rolled at $873 \mathrm{~K}$ and the latter was rolled unidirectionally at $1173 \mathrm{~K}$. Both sheets were rolled from hot rolled sheets of $5 \mathrm{~mm}$ thickness to a reduction of $77 \%$. In the cross rolling, the square specimen were rotated $\pi / 2$ at every pass. The cross rolling was finished by 6 pass and the unidirectional rolling by 4 pass. Rolled sheets were pickled and annealed in vacuum for $7.2 \mathrm{ks}$ at $1073 \mathrm{~K}$. Annealing at the high temperature is made to sharpen the preferred orientation by grain growth ${ }^{(10)}$. Grains were equiaxed in both kinds of sheets. The mean grain size was $46 \mu \mathrm{m}$ in cross rolled sheets and $50 \mu \mathrm{m}$ in unidirectionally rolled sheets.

\section{Results and Discussion}

Etch pits were produced on coarse grains formed by the strain annealed method, according to the etching procedure presented in Table 2. Photographs a through d in Fig. 1 show examples of such etch pits. Small etch pits were about $1 \mu \mathrm{m}$ and large ones were about $5 \mu \mathrm{m}$. 


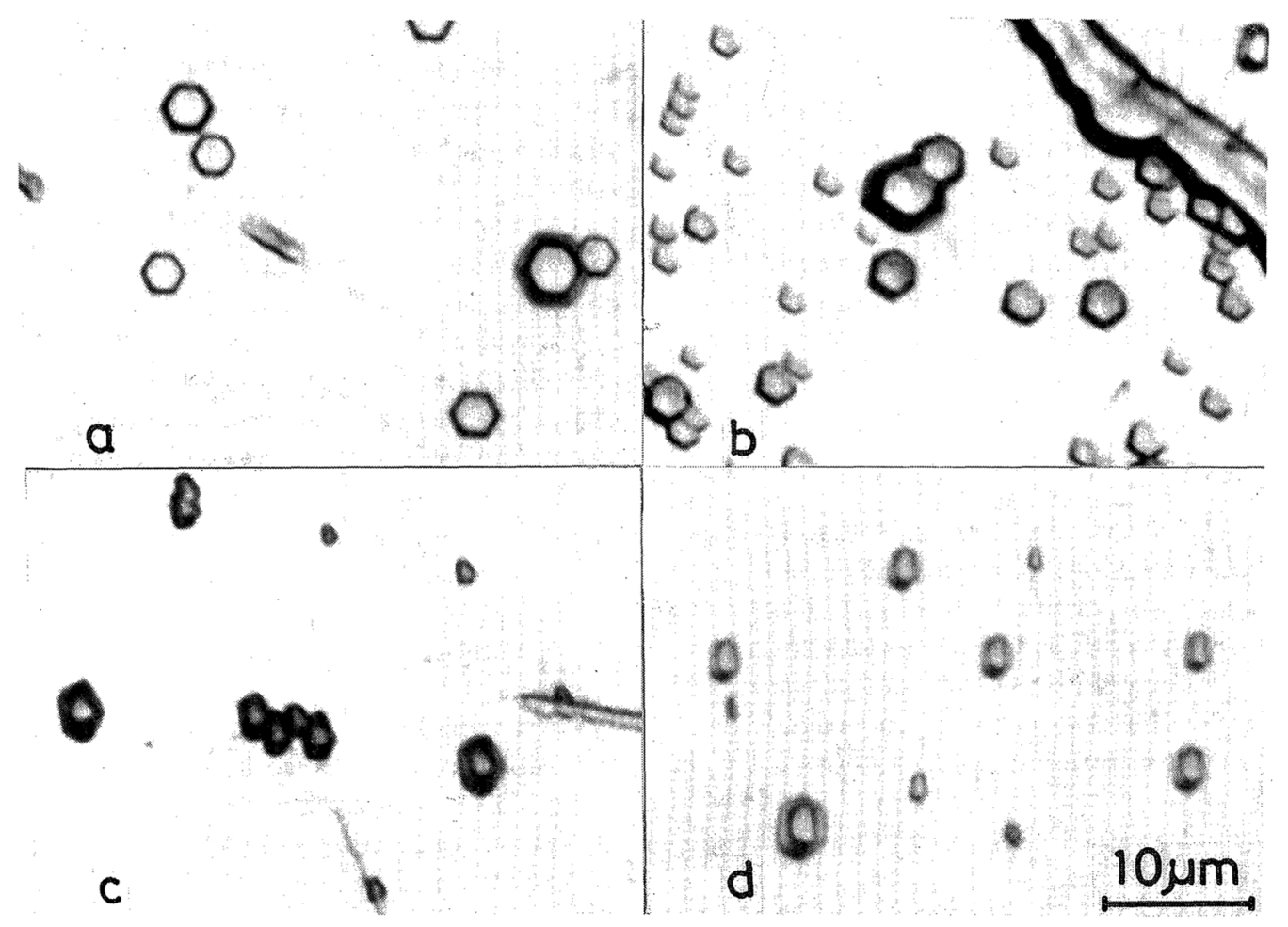

Fig. 1 Examples of etch pits on different grains.

Figure 2 shows the orientations determined from micro-Laue photographs for four grains presented in Fig. 1. Black points present (0001) poles of four grains and white ones (1010) poles. The grains $\mathrm{a}, \mathrm{b}, \mathrm{c}$ and $\mathrm{d}$ correspond to $\mathrm{a}$, $b, c$ and $d$ in Fig. 1, respectively. In the case of Fig. $1 \mathrm{a}$ and $\mathrm{b}$, the correlation between the shape of etch pits and the orientation can be easily predicted, but in the case of Fig. 1c and $d$, it is difficult to estimate the orientation intuitively. Then, the correlation between the shape of etch pits and the rotation of polyhedral models represented in Fig. 3 was considered. The basic model is the 20 -hedron composed of $\{0001\}$ plane, $\{10 \overline{1} 0\}$ plane and \{1011\} plane, as shown in Fig. 3b. The distance from the center of the polyhedron to the crystallographic plane, $R_{c}, R_{a}$ and $R_{p}$, are parameters of the shape. Figures $3 a$ and $c$ are extreme cases. Figure $3 \mathrm{a}$ shows the 8 -hedral model constituted by $\{0001\}$ plane and $\{10 \overline{1} 0\}$ plane and Fig. 3c the 14-hedral model by \{0001\} plane and $\{10 \overline{1} 1\}$ plane. When these polyhedrons are cut with an arbitrary plane, the sectional configuration corresponds to the shape of etch pits. If (0001) is taken as the reference plane, the arbitarary plane which cuts the polyhedron is represented by two angles from (0001). When the etch pits of grains in the rolled sheet are analized by polyhedral etch pits, if the reference orientation for the sheet orientation is taken, the orientation relationship between the grain and the sheet can be known immediately by three angles. (0001)[1010] was taken as the reference orientation, so that [1010], [1210] and [0001] were the rolling, transverse and sheet normal directions of the rolled sheet, respectively. Three angles are $\theta_{1}, \theta_{2}$ and $\theta_{3} . \theta_{1}$ is the angle of the rotation around the c-axis, $\theta_{2}$ around the transverse direction and $\theta_{3}$ around the sheet normal direction. Examples of these angles are represented in Fig. 2 for the grains, which orientations are determined from micro-Laue photographs. The positive sign of a rotation means a clockwise one.

The shape parameters of polyhedral models, $R_{c}, R_{p}, R_{a}$, were fixed properly, taking account 


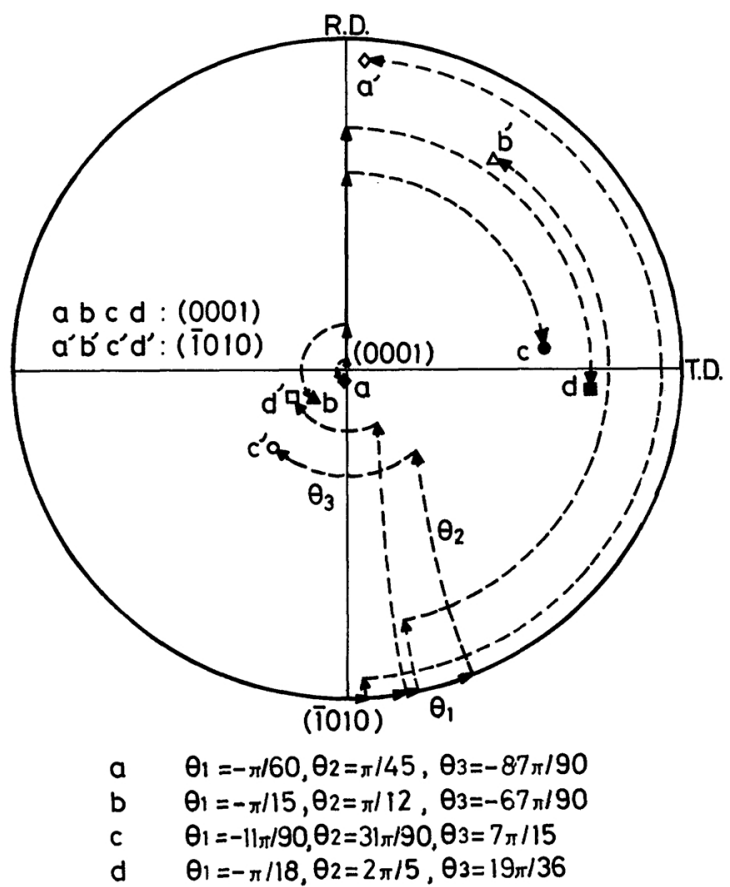

Fig. 2 The crystallographic orientations of the grains, a, $\mathrm{b}, \mathrm{c}$ and $\mathrm{d}$, shown in Fig. 1. The orientations were measured by a micro-Laue camera. The reference orientation is (0001)[10ī0].
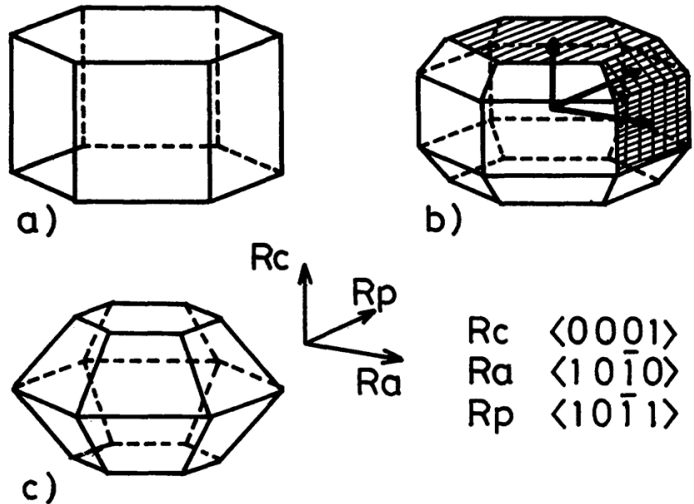

b)

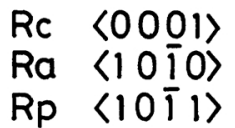

Fig. 3 The polyhedral models.

of the shape of etch pits represented in Fig. 1. Figure 4 shows the results. Every polyhedral etch pit is shape cut with the plane parallel to the sheet plane at the distance $Z=0.8 \mathrm{R}_{\mathrm{c}}$ from the center. An intersecting point of the ridge looks bright and the plane, whose normal has a large angle to the light axis, looks dark, as shown in Fig. 1c. The 8-hedral model in Fig. 4

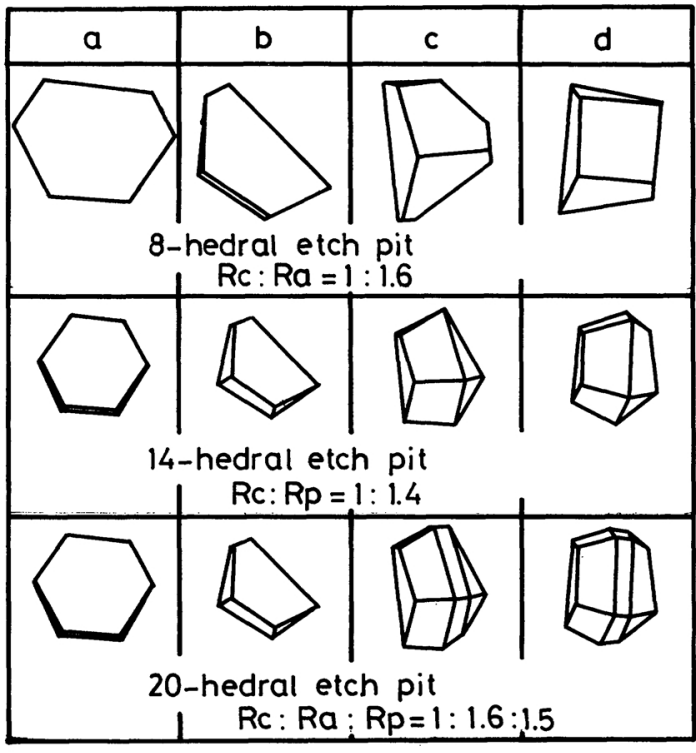

$Z=0.8 R c$

Fig. 4 The shapes of the polyhedral etch pit for the grains a, b, c and d, shown in Fig. 1 and Fig. 2. $Z$ is the distance of the cutting plane from the center of the polyhedral model.

does not coincide with the profile of etch pits. In the etch pits shown in Fig. 1d, the plane normal to the light axis looks white, but the 20hedral model cann't express this, as shown in Fig. 4. According to the comparison between Fig. 1 and Fig. 4, the 14-hedral model with the shape parameters, $R_{c}: R_{a}: R_{p}=1: 1.6: 1.4$ seems to coincide well with the shape of etch pits.

Figure 5 shows the shape of 14-hedral etch pits, the distance, $Z$, from the center to the cutting plane being altered. As etch pits in Fig. 1 become large, 14-hedral etch pits cut with the plane closer to the center correspond to them. The shapes of 14-hedral etch pits for $Z$ from $0.8 R_{c}$ to $0.4 R_{c}$ express satisfactorily etch pits of the sizes from $1 \mu \mathrm{m}$ to $5 \mu \mathrm{m}$ in Fig. 1 . If the cutting plane passes the center, $Z=0$, the difference in the shape among different orientations seems indistinct.

As stated above, etch pits revealed by the etching method presented in Table 2 can be made to correspond to the orientation of grains. The orientation can be determined easily from the 14-hedral etch pit model, $R_{c}: R_{a}: R_{p}=1: 1.6: 1.4$, in Fig. 3c. Figure 6 shows 


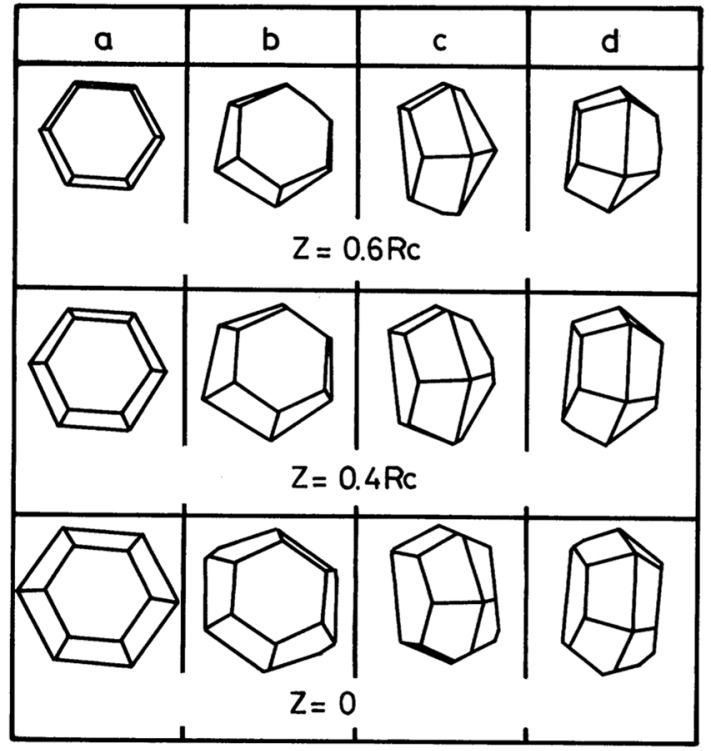

14-hedral etch pit

$R c: R p=1: 1.4$

Fig. 5 The shapes of the 14-hedral etch pit for the grains, a, b, c and d, shown in Fig. 1 and Fig. 2.

the shapes of 14-hedral etch pits changing $\theta_{1}$ and $\theta_{2} . Z=0.8 \mathrm{R}_{\mathrm{c}}$ and $\theta_{3}=0$. The orientation of individual grains in a polycrystalline aggregate can be known immediately as compared with such figures for several $Z$ values.

The textures of rolled and heat treated sheets were analized using etch pit model figures shown in Fig. 6. Figure 7 shows the (0001) pole figure for sheets annealed in vacuum for $7.2 \mathrm{ks}$ at $1073 \mathrm{~K}$ after cross rolled at $873 \mathrm{~K}$. The pole figure was determined by etch pits for 182 grains in the area $0.9 \mathrm{~mm} \times 0.6 \mathrm{~mm}$ in size. In these grains, those with the c-axis parallel to the sheet normal direction were $61 \%$, and especially those with (0001)[1010] were $23.1 \%$. Therefore, this sheet is regarded as having the preferred orientation of (0001)[1010]. Figure 8 shows the (0001) pole figure of sheets annealed in vacuum for $7.2 \mathrm{ks}$ at $1073 \mathrm{~K}$ after rolled unidirectionally at $1173 \mathrm{~K}$. The pole figure was determined by etch pits for 136 grains in the area $0.8 \mathrm{~mm} \times 0.58 \mathrm{~mm}$ in size. In these grains, those with the c-axis parallel to the sheet plane are $50 \%$. This sheet has a less preferred orientation than the cross rolled sheets. The position of the peak of the density of the basal plane

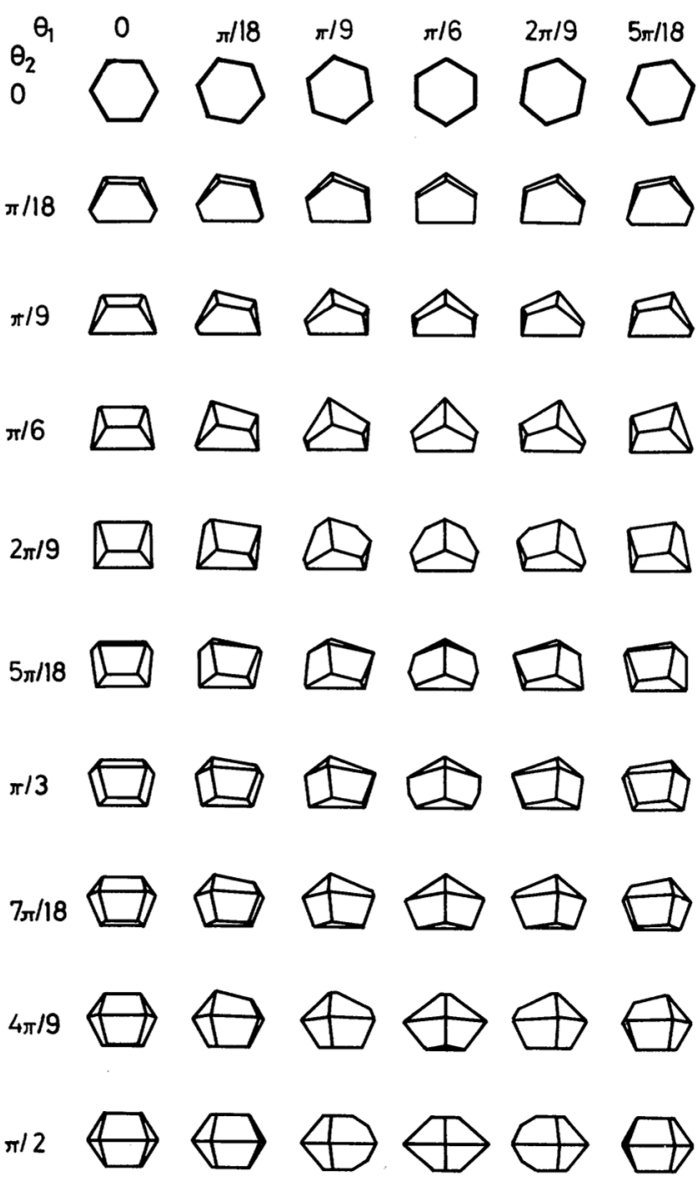

Fig. 6 14-hedral etch pits. $R_{c}: R_{p}=1: 1.4 . \quad R_{a}=R_{p} / \sin$ $(61.4 \pi / 180)=1.6 . Z=0.8 R_{c} . \theta_{3}=0$.

pole was parallel to the sheet plane and clockwise shifted about $7 \pi / 18$ from the rolling direction. Grains with (1010)[11 28$]$ are 5.1\%, those with (1010)[112 17] 4.4\% and those with (1010)[11 $\overline{2} 5] 2.9 \%$. Grains with orientations ranging from $(\operatorname{lmn} 0)[11 \overline{2} 5]$ to $(\operatorname{lmn} 0)[11 \overline{2} 17]$ are $19.8 \%$ and those from $(10 \overline{1} 0)[11 \overline{2} 5]$ to (1010)[11 17 17] are $12.5 \%$. Therefore, this sheet has on the whole the preferred orientation ex-

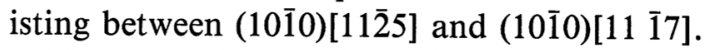

Figure 9 shows the orientation relation among all neighboring grains in the field of view. The mechanical properties of pure titanium are extremely anisotropic between the direction parallel to the basal plane and that normal to it, because it has the hep structure and slip directions of all slip systems are parallel to the basal plane. Therefore, the orien- 


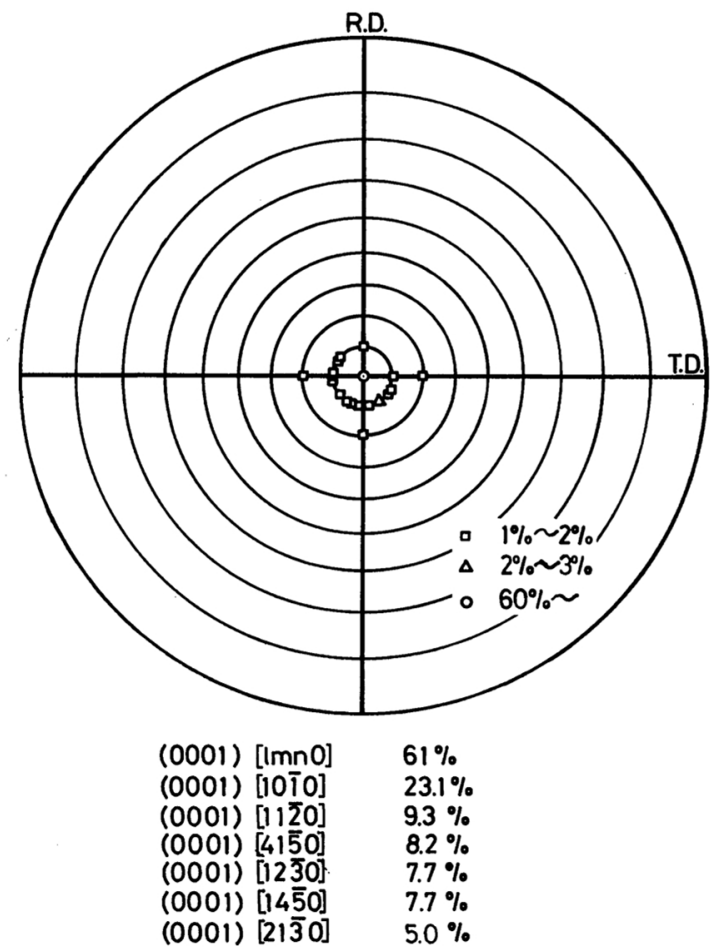

Fig. 7 The (0001) plane pole figure for the sheet cross rolled at $873 \mathrm{~K}$. The frequency is calculated from the number of the grains having the etch pits on the surface.

tation difference of the c-axis between neighboring grains was treated. Figure 9(a) shows the results from the cross rolled sheets. The frequency of the orientation difference of the c-axis between neighboring grains with angles between 0 and $\pi / 18$ is over $80 \%$. In the sheets rolled unidirectionally at $1173 \mathrm{~K}$, the orientation difference of the c-axis between neighboring grains is broadly distributed in the whole range from 0 to $\pi / 2$, as in Fig. $9(\mathrm{~b})$.

Figure 10 shows the orientation relation when only the grains with the preferred orientation are considered. The grains with (0001) [1010] in cross rolled sheets and those with orientations from (1010)[1125] to $(10 \overline{1} 0)[11 \overline{2}$ 17] in unidirectionally rolled sheets were treated. As compared with Fig. 9, the frequency of the orientation difference ranging from 0 to $\pi / 18$ increases both in cross rolled sheets and unidirectionally rolled sheets. It increases from $80.2 \%$ to $93.2 \%$ in cross rolled sheets and from $10.1 \%$ to $24.5 \%$ in unidirectionally rolled sheets.

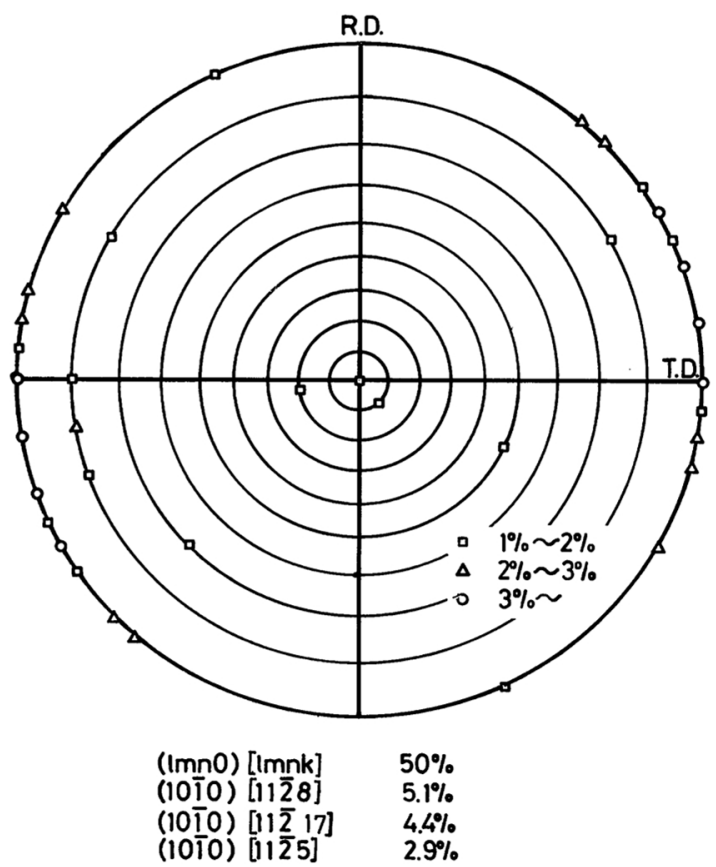

Fig. 8 The (0001) plane pole figure for the sheet rolled unidirectionally at $1173 \mathrm{~K}$. The frequency is calculated from the number of the grains having the etch pits on the surface.

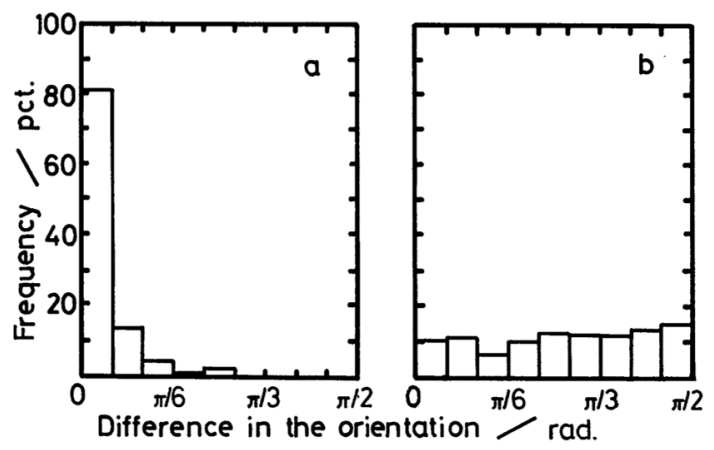

Fig. 9 The frequency of the difference in the orientation of the c-axis between the neighboring grains. (a) The sheet cross rolled at $873 \mathrm{~K}$. (b) The sheet rolled unidirectionally at $1173 \mathrm{~K}$.

In the case of cross rolled sheets, the existence probability of grains with the preferred orientation, (0001)[1010], is high, $23.1 \%$, and the orientation difference ranging from 0 to $\pi /$ 18 between the c-axis of them and that of their neighboring grains is high, 93.2\%. Therefore, this sheets are expected to have the mechanical properties close to those of a single crystal in spite of polycrystalline aggregate. In sheets 


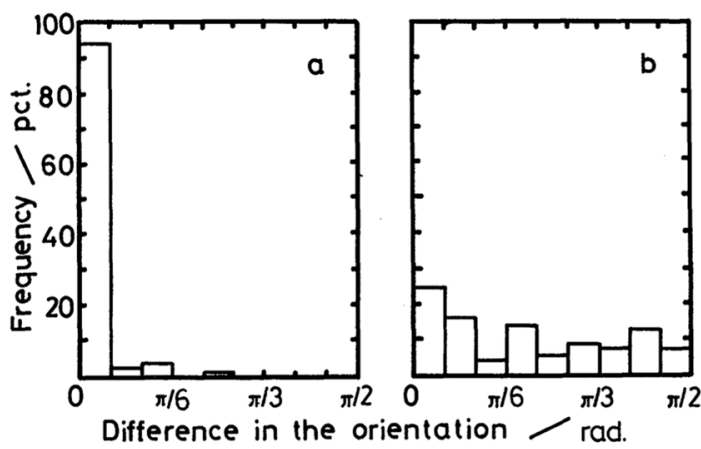

Fig. 10 The frequncy of the difference in the orientation of the c-axis between the grains having the preferred orientation and their neighboring grains. (a) The sheet cross rolled at $873 \mathrm{~K}$. The preferred orientation is $(0001)$ [1010]. (b) The sheet rolled unidirectionally at $1173 \mathrm{~K}$. The preferred orientation is form (1010)[11 $\overline{2} 5]$ to (1010[11̄ 17].

rolled unidirectionally at $1173 \mathrm{~K}$, grains with the c-axis parallel to the sheet plane are $50 \%$, but the orientation difference of the c-axis between neighboring grains is broadly distributed in the whole range from 0 to $\pi / 2$. For grains with the preferred orientation, the orientation difference is also distributed from 0 to $\pi / 2$, although the orientation difference from 0 to $\pi / 18$ increases to $24.5 \%$. Therefore, this textured sheets are expected to suffer more plastic constraint by the orientation difference between neighboring grains than cross rolled sheets.

\section{Conclusion}

In this experiment, the procedure to produce oriented etch pits on a commercially pure titanium sheet was presented, and the feature of the orientation distribution of grains, containing the orientation relation between neighboring grains, was analized by the etch pit method. The shape of etch pits is coincident with that introduced from a rotation of the 14-hedral model, having shape parameters, $R_{c}: R_{a}: R_{p}=1: 1.6: 1.4$. The orientation of grains with etch pits is easily known by 14-hedral etch pits.

Using such oriented etch pits, the features of the orientation distribution in two differently textured titanium sheets were examined. In sheets cross rolled at $873 \mathrm{~K}$, the preferred orien- tation is (0001)[1010] and the frequency ranging from 0 to $\pi / 18$ of the orientation difference of the c-axis between grains with the preferred orientation and their neighboring grains, is 93.2\%. In sheets unidirectionally rolled at $1173 \mathrm{~K}$, the preferred orientation ranges from (1010)[11 $\overline{2} 5]$ to $(10 \overline{1} 0)[11 \overline{2} 17]$, and the orientation difference of the c-axis between neighboring grains is distributed uniformly in the whole range from 0 to $\pi / 2$. These different distributions of the orientation difference between neighboring grains suggest that the representation of a texture of titanium sheets only by the preferred orientation is insufficient. Especially, in the case of titanium whose single crystal has high anisotropy in the mechanical properties, the deformation behavior is expected to be influenced by the orientation relation between neighboring grains.

\section{Acknowledgments}

The authors would like to thank Dr. K. Ikeda of RIISOM, Tohoku University for valuable advice. This work was supported by Grant-in-Aid for Scientific Research from The Ministry of Education, Science and Culture, Japan.

\section{REFERENCES}

(1) Haward T. Clark, Jr.: Trans. AIME, 188 (1950), 1154.

(2) J. H. Keeler and A. H. Geisler: J. Metals, 8 (1956), 80.

(3) A. J. Hatch: Trans. Met. Soc. AIME, 233 (1965), 44.

(4) A. Hasegawa, T. Nishimura and M. Fukuda: Titanium and Titanium Alloys, vol. 3 (1976)/1982), 1947.

(5) D. V. Wilson, W. T. Roberts and P. M. B. Podrigues: Met. Trans., 12A (1981), 1595.

(6) D. V. Wilson, W. T. Roberts and P. M. B. Podrigues: Met. Trans., 12A (1981), 1603.

(7) P. K. Sengupta, W. T. Roberts and D. V. Wilson: Met. Tech., 8 (1981), 171.

(8) Metal Technology Series 3, Collection of Photometallography for Light Metals, ed. by $\mathrm{O}$. Izumi, S. Morozumi and M. Terasawa under the supervision of S. Koda, Japan Institute of Metals, (1972), p. 144.

(9) Y. Kinoshita and S. Takeda: Bulletin of the Japan Institute of Metals, 18 (1979), 642.

(10) S. Kohara: Proceedings of Symposiumm Deformability of Titanium and it's Alloys, Japan Institute of Metals, (1981), p. 19. 